\title{
MESOFAUNA EDÁFICA EM ÁREA DE OCORRÊNCIA DA ESPÉCIE Byrsonima gardneriana A. Juss, NA CAATINGA ALAGOANA
}

\author{
Ana Beatriz da Silva', Élida Monique da Costa Santos2, Danúbia Lins Gomes³, João Gomes da Costa4, \\ Kallianna Dantas Araujo ${ }^{5}$ \\ 1'Discente do PPGG-Instituto de Geografia, Desenvolvimento e Meio Ambiente, Universidade Federal de Alagoas, CEP 57072-970, \\ Maceió-Alagoas, E-mail: anabtrizsilva@gmail.com \\ ${ }^{2}$ Mestra em Geografia pelo PPGG/IGDEMA/UFAL, E-mail: elida_monique2@hotmail.com \\ ${ }^{3}$ Discente do PPGDIBICT/ICBS/UFAL, E-mail: dlinsgomes@yahoo.com.br \\ 4Docente do PPGAA/UFAL/Campus Arapiraca, E-mail: joão-gomes.costa@embrapa.br \\ ${ }^{5}$ Docente do PPGG/IGDEMA/UFAL,E-mail: kallianna.araujo@igdema.ufal.br
}

\begin{abstract}
RESUMO: Os organismos da mesofauna edáfica exercem no solo funções importantes e mantem interação com as espécies vegetais. Objetivou-se avaliar a riqueza, abundância e diversidade dos organismos da mesofauna edáfica que atuam em ambiente com ocorrência da espécie Byrsonima gardneriana A. Juss, em Olho D’Água do Casado, Semiárido Alagoano. A pesquisa foi realizada em Agosto de 2017. Foram selecionados cinco pontos próximos as matrizes da espécie $B$. gardneriana para avaliação da mesofauna edáfica por meio de anéis metálicos instalados na bateria de extratores Berlese-Tullgren modificada, por 96 horas, para extração dos organismos. A mesofauna capturada foi quantificada e identificada com auxílio de lupa binocular e chave de identificação, sendo avaliada de forma quantitativa (abundância e riqueza de grupos) e qualitativa (índice de diversidade de Shannon). Realizou-se medição de altura das matrizes de B. gardneriana para relacionar com a mesofauna edáfica. Os grupos taxonômicos da mesofauna edáfica mais abundantes em área de ocorrência de B. gardneriana são Collembola e Acarina; Os organismos registrados apresentam-se como os possíveis agentes influenciadores do desenvolvimento das matrizes de $B$. gardneriana; As matrizes de $B$. gardneriana com maior altura, proporcionam microhabitats mais propícios ao desenvolvimento da mesofauna edáfica, refletindo no equilíbrio do ambiente.
\end{abstract}

Palavras-chave: Semiárido, Espécie nativa, Fauna do solo.

\section{EDAPHIC MESOFAUNA IN THE AREA OF OCCURRENCE OF THE SPECIES Byrsonima gardneriana A. Juss, CAATINGA ALAGOANA}

ABSTRACT: Edaphic mesofauna organisms exert important functions in soil and maintain interaction with plant species. The aim of this study was to evaluate the richness, abundance and diversity of soil edaphic mesofauna organisms which acts in an environment with occurrence of Byrsonima gardneriana A. Juss in Olho D'Água do Casado, Alagoas semiarid. The research was conducted in August 2017. It was selected five points near $B$. gardneriana matrices for evaluating edaphic mesofauna by metal rings installed in modified BerleseTullgren extractor battery, for 96 hours, to extract the organisms. Mesofauna captured was quantified and identified with aid of a binocular loupe glass and identification key, being evaluated quantitatively (abundance and group richness) and qualitative (Shannon diversity index). It was conducted a measurement of height for $B$ gardneriana matrices relate to the soil mesofauna. The most abundant taxonomic groups of the soil mesofauna in the area of occurrence of $B$. gardneriana are Collembola and Acarina; Registered organisms present as possible agents influencing the development of $B$. gardneriana matrices; $B$. gardneriana matrices with greater height, provide microhabitats more propitious to the development of edaphic mesofauna, reflecting in environment balance.

Keywords: Semiarid, Native species, Soil Fauna. 


\section{INTRODUÇÃO}

A mesofauna edáfica é composta por organismos que possuem comprimento entre 0,2 e 2,0 mm, responsáveis pela decomposição do material vegetal, ciclagem de nutrientes e regulação dos processos biológicos do solo (BERUDE et al., 2015), imprescindíveis para o desenvolvimento da planta, somado a água e nutrientes presentes no solo, que são necessários neste processo (NABORS, 2012).

Das espécies vegetais endêmicas da Caatinga, Byrsonima gardneriana A. Juss (Murici) se destaca pela ampla distribuição na Microrregião Geográfica Alagoana do Sertão do São Francisco, notadamente em Olho D'Água do Casado e seus frutos apresentam potencial para ser explorado economicamente em forma de flau, suco, doce, dentre outros (SANTOS, 2016). Sendo necessário o conhecimento dos organismos da mesofauna edáfica que ocorrem na área de abrangência desta espécie.

Objetivou-se avaliar a riqueza, abundância e diversidade dos organismos da mesofauna edáfica presente em ambiente com ocorrência da espécie Byrsonima gardneriana A. Juss em Olho D'Água do Casado, Semiárido Alagoano.

\section{MATERIAL E MÉTODOS}

A pesquisa foi realizada em área experimental de Caatinga em Olho D'Água do Casado, Alagoas, localizado nas coordenadas geográficas $10^{\circ} 03^{\prime} 30^{\prime \prime S}$ e $36^{\circ} 49^{\prime} 00^{\prime \prime} \mathrm{W}$, na altitude 230 m (ALAGOAS, 2015). O clima da área de estudo é Tropical Semiárido (BSh), segundo a classificação de Köppen (LIMA, 1977).

A área de Caatinga estudada possui 1 ha $e$ encontra-se circundada por afloramento rochoso. A amostragem da mesofauna edáfica foi realizada em agosto de 2017 em cinco pontos de coleta, próximo as matrizes da espécie $B$. gardneriana.

As coletas foram feitas por meio de anéis metálicos com diâmetro de $4,8 \mathrm{~cm}$ e altura de $5 \mathrm{~cm}$, sendo retiradas amostras de solo e serapilheira com ajuda de pá de jardinagem e estes foram envolvidos em tecidos TNT e filó de cor branca, amarrados com ligas de borracha (Figura 1A).

Os anéis foram instalados na bateria de extratores Berlese-Tullgren modificada para extração de organismos do solo, por um período de 96 horas (Figura 1B) (SWIFT et al., 1979).

Figura 1. Coleta da mesofauna edáfica com anéis metálicos $(A)$, os quais foram instalados na bateria de extratores Berlese-Tullgren modificada (B).

A

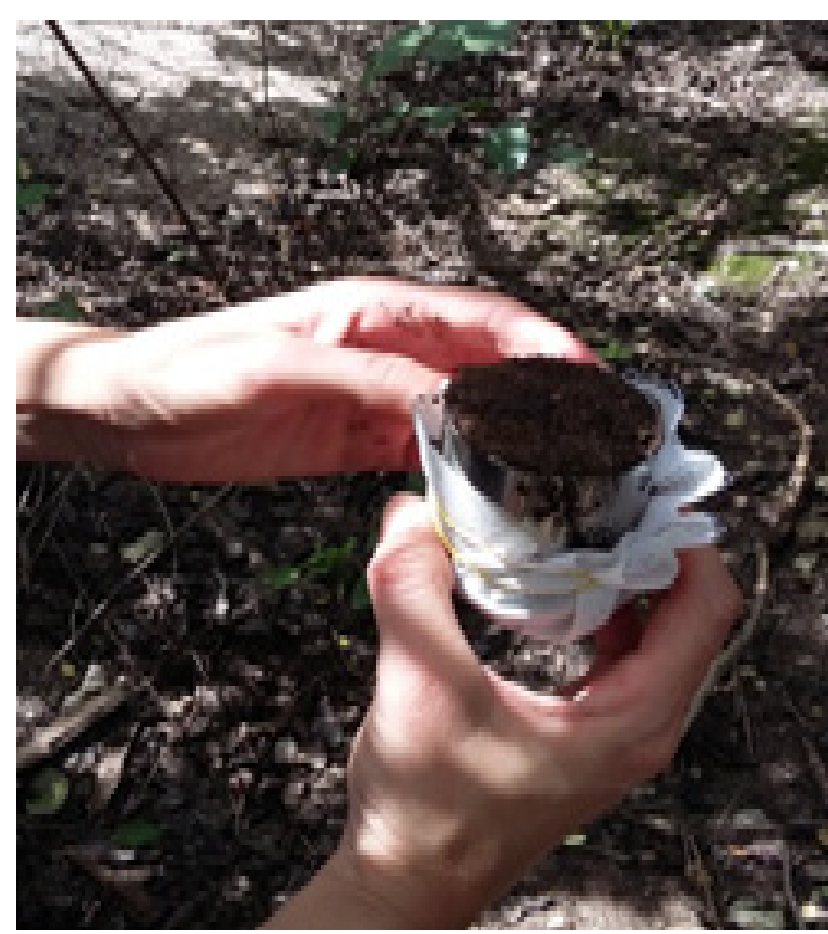

B

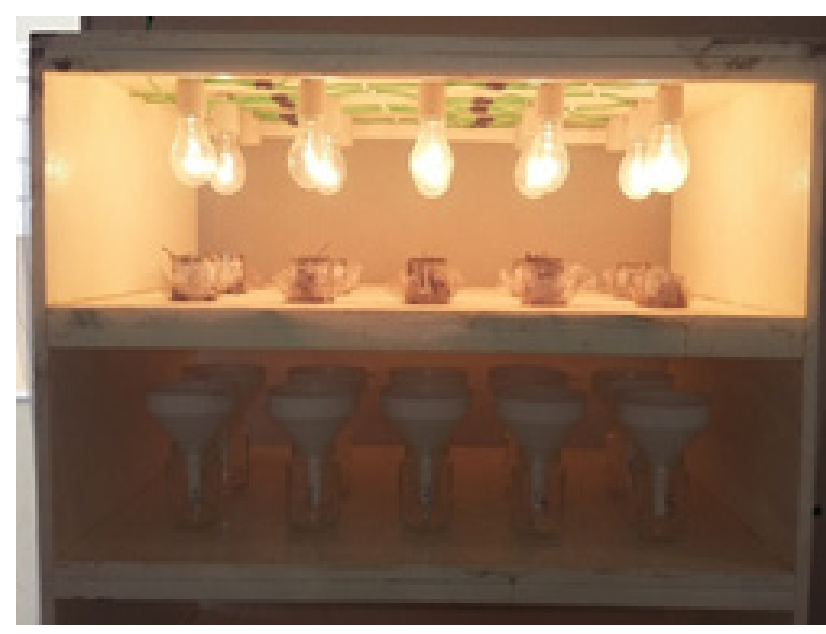

A mesofauna capturada com comprimento entre 0,2 e 2,0 mm foi quantificada e identificada com auxílio de lupa binocular (Figura 2) e chave de identificação de Triplehorn e Jonnson (2011) no Laboratório de Ecogeografia e Sustentabilidade Ambiental-LABESA/ IGDEMA/UFAL. 
Figura 2. Contagem e identificação dos organismos da mesofauna edáfica em lupa binocular.

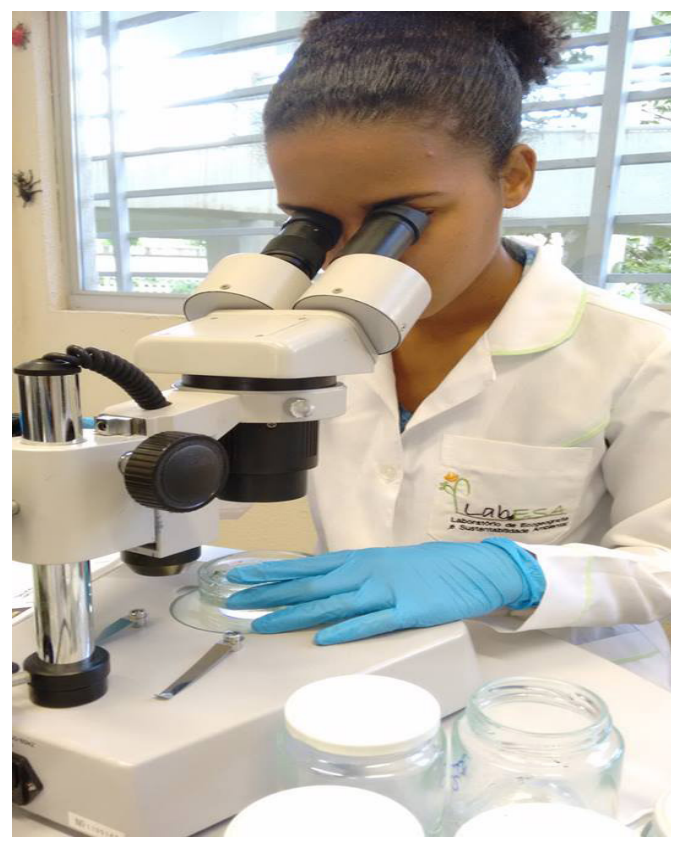

Para a análise quantitativa foi avaliada abundância e riqueza de grupos taxonômicos e na análise qualitativa foi aplicado o Índice de Diversidade de Shannon $(H)$, pela equação: $H=-\sum$ pi.logpi, em que: $\mathrm{pi}=\mathrm{ni} / \mathrm{N}$; ni=densidade de cada grupo; $\mathrm{N}=\Sigma$ da densidade de todos os grupos (BEGON et al., 1996).

Foram realizadas medidas de altura de cada matriz de $B$. gardneriana com auxílio de régua graduada, para relacionar com a mesofauna edáfica (Figura 3).

Figura 3. Medida de altura da espécie $B$. gardneriana.

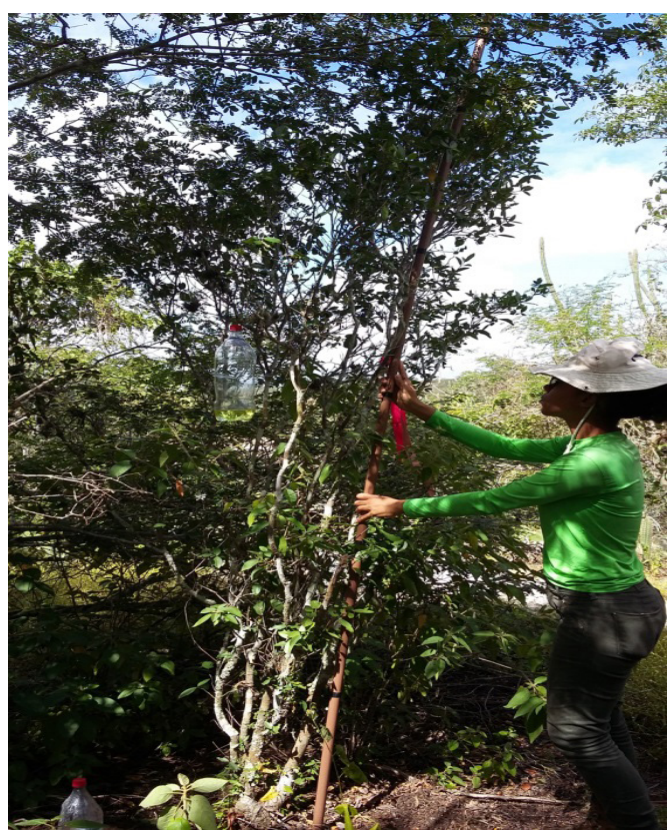

Foram registrados 101 organismos da mesofauna, distribuídos em três grupos taxonômicos (Figura 4) (Figura 5). Os grupos mais dominantes foram Collembola (55 ind.) e Acarina (19 ind.) (Figura 4).

Segundo Rieff (2010), as ordens Acarina e Collembola dominam em abundância e diversidade, compondo de 72 a $95 \%$ do número total de microártropodes, ocorrendo dominância destes grupos nas matrizes de $B$. gardneriana $A$. Juss.

Figura 4. Abundância e riqueza de grupos taxonômicos próximo as matrizes da espécie $B$. gardneriana, em Olho D’Água do Casado, Alagoas.

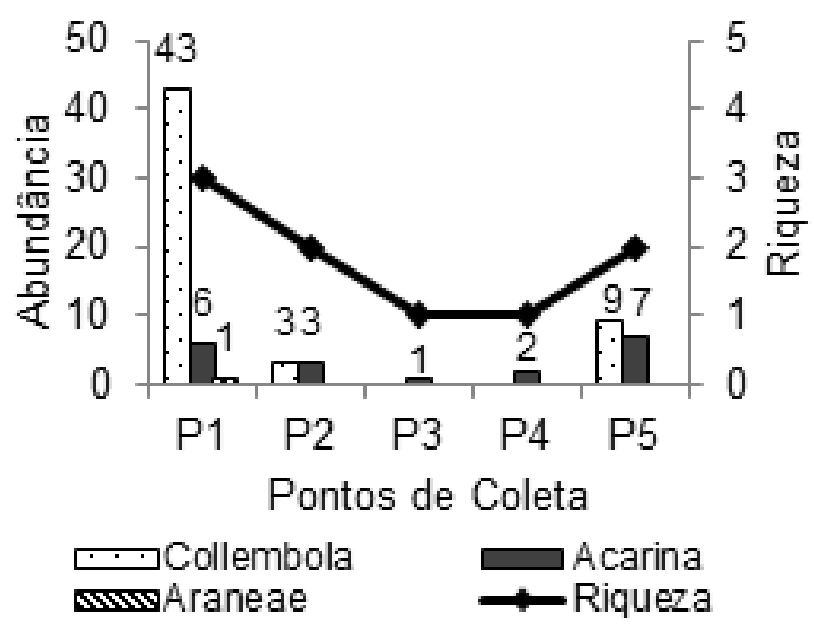

Figura 5. Grupos taxonômicos da mesofauna edáfica: Collembola (A), Acarina (B) e Araneae (C), registrados próximos às matrizes de $B$. gardneriana.
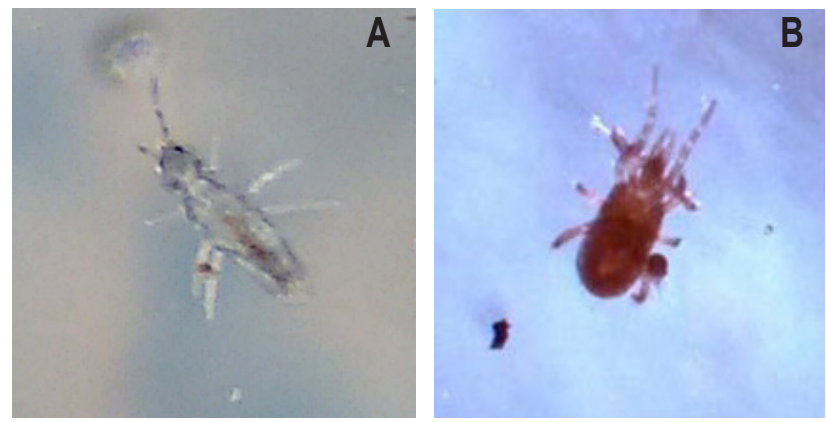

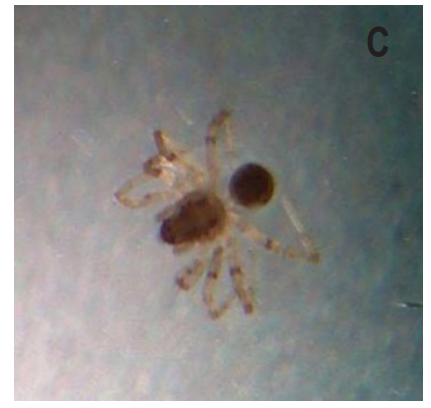


Observou-se uma tendência da maior riqueza e abundância da mesofauna edáfica ocorrer próximo das matrizes de $B$. gardneriana de maior porte (Figuras 4 e 6), sendo composto em sua maioria por Collembola, que tem preferência por ambientes mais úmidos e sombreados, cuja ação no solo influencia o desenvolvimento vegetal, o transporte de fungos e bactérias benéficas para a planta (UFV, 2018).

Figura 6. Riqueza $(A)$ e abundância $(B)$ da mesofauna relacionada com a altura $(\mathrm{m})$ das matrizes da espécie B. gardneriana, em Olho D’Água do Casado, Alagoas.
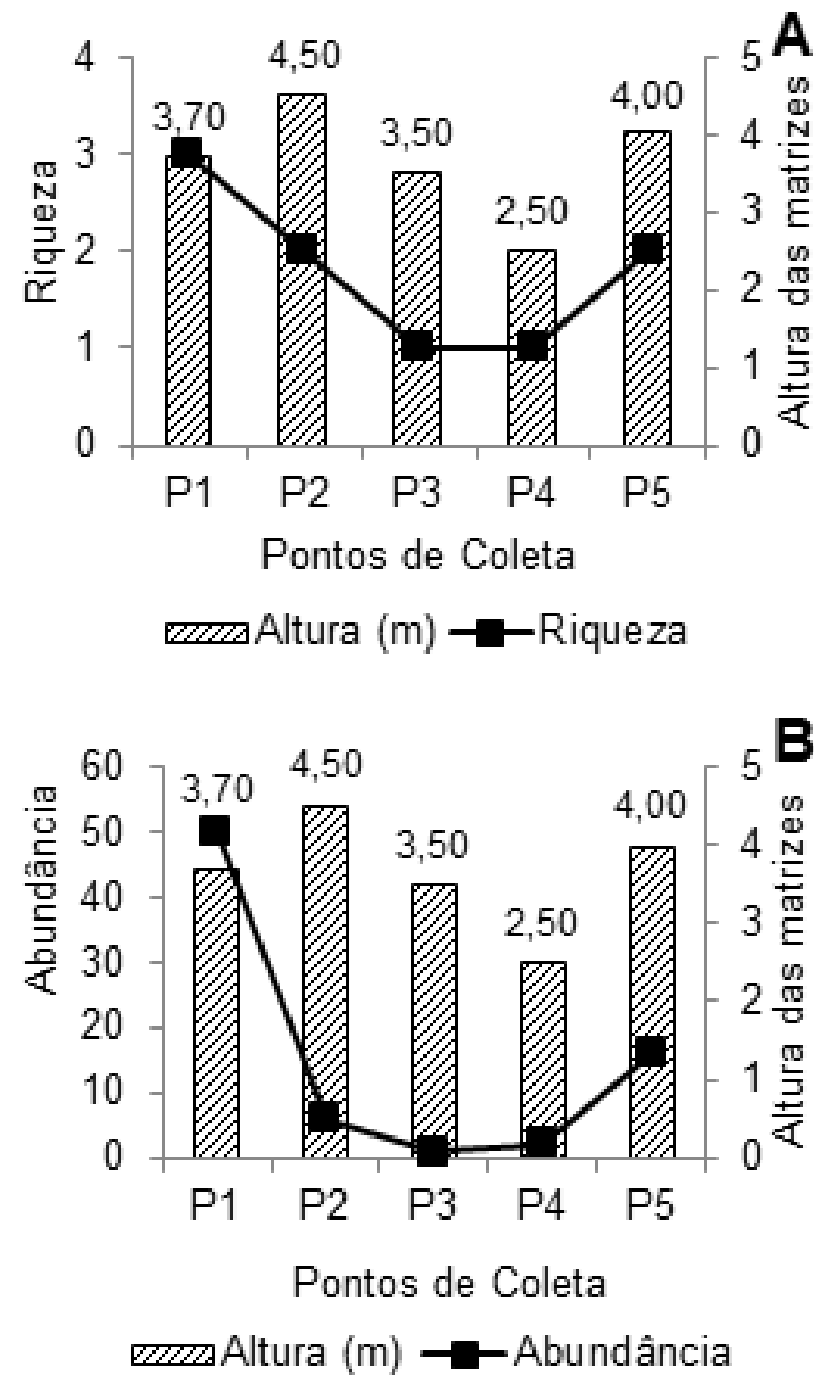

A diversidade entre os grupos taxonômicos avaliados foi menor para Collembola $(0,13)$ que apresentou-se como o mais dominante, em virtude do índice de Shannon potencializar as espécies raras com maior valor para Araneae $(1,88)$ com baixa dominância, (Figura 7).
Figura 7. Índice de Diversidade de Shannon $(H)$, dos grupos taxonômicos registrados próximos as matrizes de B. gardneriana, em Olho D'Água do Casado, Alagoas.

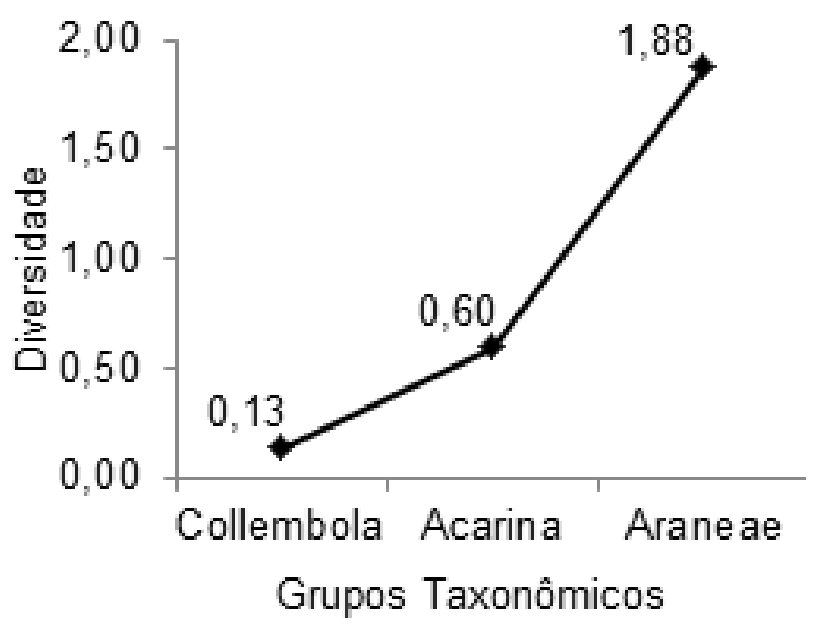

Deste modo contatou-se que as matrizes de $B$. gardneriana propiciam microhabitats que influenciam o crescimento, desenvolvimento e sobrevivência dos organismos da mesofauna edáfica.

\section{CONCLUSÕES}

Os grupos taxonômicos da mesofauna edáfica mais abundantes em área de ocorrência de $B$. gardneriana são Collembola e Acarina; Os organismos registrados apresentam-se como os possíveis agentes influenciadores do desenvolvimento das matrizes de $B$. gardneriana; As matrizes de $B$. gardneriana com maior altura, proporcionam microhabitats mais propícios ao desenvolvimento da mesofauna edáfica, refletindo no equilíbrio do ambiente.

\section{AGRADECIMENTOS}

Ao Laboratório de Ecogeografia e Sustentabilidade Ambiental-IGDEMA/UFAL, pela identificação dos grupos taxonômicos da mesofauna edáfica.

\section{REFERÊNCIAS}

ALAGOAS. Perfil municipal: Olho D’Água do Casado. 3. ed. Maceió: SEPLANDE/AL, 2015. 24 p.

BEGON, M.; TOWNSEND, C. R.; HARPER, J. L. Ecology: individuals, populationsand communities. 3. ed. Oxford: Blackwell Science, 1996. 92 p. 
BERUDE, M. C. et al. A mesofauna do solo e sua importância como bioindicadora. Enciclopédia Biosfera, 2015, 11, 14-28.

LIMA, I. F. Fundamentos geográficos do meio físico do Estado de Alagoas. 1. ed. Maceió: Governo do Estado de Alagoas/SEPLANDE/SUDENE,1977. 106 p. (Série: Estudo de Regionalização).

NABORS, M. W. INTRODUÇÃO À BOTÂNICA. 1. ED. SÃO PAULO: ROCA, 2012. 646 P.

RIEFF, G. G. MONITORAMENTO DE ÁCAROS E COLEMMBOLOS COMO INDICADORES DE QUALIDADE DO SOLO. 2010. 59 F. DISSERTAÇÃO (MESTRADO EM CIÊNCIA DO SOLO)-FACULDADE DE AGRONOMIA, UNIVERSIDADE FEDERAL DO RIO GRANDE DO SUL, PORTO ALEGRE, 2010.
SANTOS, E. M. DA C. IMPORTÂNCIA SOCIOECONÔMICA DA ESPÉCIE BYRSONIMA GARDNERIANA A. JUSS (MURICI) COMO ALTERNATIVA DE RENDA COMPLEMENTAR PARA A POPULAÇÃO DO SEMIÁRIDO ALAGOANO. 2016. 140 F. DISSERTAÇÃO (MESTRADO EM GEOGRAFIA)INSTITUTO DE GEOGRAFIA, DESENVOLVIMENTO E MEIO AMBIENTE, UNIVERSIDADE FEDERAL DE ALAGOAS, MACEIÓ, 2016.

SWIFT, M. J.; HEAL, O. W.; ANDERSON, J. M. DECOMPOSITION IN TERRESTRIAL ECOSYSTEMS: STUDIESINECOLOGY.5. ED. OXFORD: BLACKWELL SCIENTIFIC, 1979. 238 P.

TRIPLEHORN, C. A.; JONNSON, N. F. ESTUDO DOS INSETOS. 1. ED. SÃO PAULO: CENGAGE LEARNING, 2011. $809 \mathrm{P}$.

UFV - UNIVERSIDADE FEDERAL DE VIÇOSA. ENTOMOLOGIA: VIÇOSA: UFV, 2018. DISPONIVEL EM <WWW.INSECTA.UFV.BR>. ACESSO: 28 DE MAR. 2018, 16:32:54. 